\title{
Extended Phylogeny of the Equine Arteritis Virus Sequences Including South American Sequences
}

\author{
Germán Ernesto Metz ${ }^{a, b}$ Giselle Paula Martín Ocampos ${ }^{a, b}$ \\ María Soledad Serena ${ }^{a, b}$ Sabrina Eliana Gambaro ${ }^{a}$ Edgardo Nosetto ${ }^{a}$ b \\ María Gabriela Echeverría a, b \\ a Virology, and ${ }^{b}$ CONICET (Scientific Research Council), Faculty of Veterinary Sciences, National University of \\ La Plata, La Plata, Argentina
}

\section{Key Words}

Equine arteritis virus - South American equine arteritis virus sequences • Equine arteritis, Argentina • Phylogenetic analysis

\begin{abstract}
Objective: To perform genetic analysis of the ORF5 of equine arteritis virus (EAV) may provide new insights into the genetic evolution and origin of the Argentinean EAV sequences. Methods: A total of 76 sequences were analyzed by neighbor joining (NJ), maximum parsimony and maximum likelihood algorithms. The analysis of the selective pressures was performed using the Tajima's test. Results: The trees showed similar topologies. Two clades were identified: the first clade was formed by strains isolated mainly from a donkey, whereas the second clade presented four large groups from different geographic regions exclusively from Equus caballus. In this clade, we identified a group formed by South African and another one by South American and European sequences. In the latter, the monophyletic group was formed by seven Argentinean sequences. In the NJ tree, we identified a group formed by six Argentinean sequences. The Tajima's test showed a D value of 1.73663 , indicating that the sequences analyzed follow a neutral evolution model. Con-
\end{abstract}

clusion: We concluded that the Argentinean sequences have a paraphyletic origin and that the fixation of point mutation might follow the neutral model evolution; however, we identified purifying pressures that may be involved in the differentiation of the EAV sequences.

Copyright $\odot 2010$ S. Karger AG, Basel

\section{Introduction}

Equine arteritis virus (EAV) is a positive-stranded RNA virus, first isolated from fetal lung tissue during an outbreak of respiratory disease and abortion in Standardbred horses in Bucyrus, Ohio, USA, in 1953 [1]. EAV has been classified in the family Arteriviridae, Order Nidovirales, and grouped together with lactate dehydrogenase-elevating virus, porcine reproductive and respiratory syndrome (PRRS) virus, and simian hemorrhagic fever virus [2]. The EAV genome is $12.7 \mathrm{~kb}$ long and has nine open reading frames (ORFs), named $1 \mathrm{a}, 1 \mathrm{~b}, 2 \mathrm{a}, 2 \mathrm{~b}$, $3,4,5,6$ and 7, encoding structural and non-structural proteins [3]. ORF5, which encodes the GP5 protein, is the most variable ORF of the EAV genome and is located between nucleotides (nt) 11,146 and 11,913 [4]. This envelope glycoprotein expresses neutralization determinants

\section{KARGER}

Fax +4161306 1234 E-Mail karger@karger.ch www.karger.com (c) 2010 S. Karger AG, Basel

0300-5526/11/0541-0030\$38.00/0

Accessible online at:

www.karger.com/int
María Gabriela Echeverría

Faculty of Veterinary Sciences

National University of La Plata, 60 and 118

La Plata 1900 (Argentina)

Tel./Fax +54 221425 7980, E-Mail gecheverria@fcv.unlp.edu.ar 
of EAV and, together with the M protein, forms disulfidelinked heterodimers that generally induce neutralizing antibodies [3, 5]. EAV spreads by respiratory and venereal routes $[6,7]$. In most cases, EAV causes only subclinical infection, but it could cause death of foals, abortions of mares and mild clinical respiratory symptoms in adult horses [3]. Adult stallions may develop chronic infection and become a major source of infection over a long period of time by spreading the virus via semen, acting as a natural reservoir of the disease [8]. The prevalence of EAV-infected horses in Argentina is relatively low (0.5\%) [9]. The isolates of EAV in our country were carried out in 2001, 2002 and 2007 from seropositive imported stallions located in three farms with high seroprevalence of EAV. Based on the partial sequences of ORF5, the Argentinean EAV strains and sequences are grouped with the European EAV strains [10-13].

Genetic variability among EAV strains has been studied by sequence analysis of ORF5, which grouped worldwide strains into two clades: North American and European lineages [14-17]. The fact that some viruses from the North American lineage were isolated in Europe and some viruses from European lineage were isolated in North America, reflects the carriage of viruses between the two continents, which results mainly from the trade of infected stallions or EAV-infective semen. Since the same situation occurs in Argentina, the genetic comparison of the ORF5 gene sequences of the Argentine isolated virus and the analysis of the new sequences described here may provide new insights into the genetic evolution and origin of the Argentinean EAV sequences. In this work, 68 sequences available in the GenBank were used, in order to provide a most comprehensive phylogeny of the Argentinean EAV sequences.

\section{Materials and Methods}

\section{Viral Strains and Sequences}

Five strains from semen samples isolated in our laboratory were used. The first strain, named LP01, was isolated in 2001 from an imported seropositive stallion held in isolation at a military riding breeding farm in Tandil City, in the Province of Buenos Aires, with high seroprevalence of EAV [10]. This farm breeds and trains about 700 horses for jumping and other sports (Farm A). The strains LP02/P, LP02/C and LP02/R were isolated in 2002 from stallions from another farm also located in Buenos Aires Province. These three strains were obtained from three Warmblood stallions (registered as Zangersheide branded). At the time of the study, this farm, which also breeds and trains jumping horses, had approximately 280 horses, including stallions, mares, donor mares and foals (Farm B) [11]. Circumstantial evidence sug- gested that the EAV dissemination inside the farm was initiated by one of these three stallions imported to this farm from Europe in 2001. These animals had no evidence of clinical disease but soon after the new stallions arrived, other stallions, mares and foals on the farm became seropositive to EAV with an estimated prevalence of $45 \%[11,12]$. At that moment, the animal health control authorities closed both farms in order to stop the spreading of the virus caused by the trade of horses. The strain LT-LPARG was isolated from a testicle stored for 7 years at $-20^{\circ}$, which belonged to the presumably first stallion infected with equine arteritis in Argentina. The stallion was brought to Argentina from Europe in 1992 and was detected positive for EAV by virus neutralization in 1998 in our laboratory. We do not have any report about virus isolation or test mating of this horse from any other Argentinean reference laboratory. This stallion (Warmblood registered in Zangersheide) was introduced to another farm of jumping horses before Argentinean EAV control measures took place. There is not more history available from this farm. It was closed some years ago (Farm C) [12]. It is very important to remark that in Argentina exists an intensive national and international movement of horses, and international transportation of semen for insemination. For sport competitions, there was movement of the stallions between the three farms mentioned above. This fact suggested that horizontal transmission between stallions can occur and could be the reason for the positive serology. Horizontal transmission has been reported by Guthrie et al. [18]. The lack of any EAV import control measures in our country before 1994, minimal knowledge of the disease and economic reasons permitted the import of stallions only for sports purposes. Since 1994, new measures for EAV control have been introduced in Argentina without vaccination [12]. Virus isolation was performed in the RK13 cell line according to international OIE protocol [19]. On the other hand, three new sequences of EAV obtained from three archive semen samples that were negative by virus isolation were included in this work: RO-LP-ARG, from a horse from Farm A, and RZ-LP-ARG and KB-LP-ARG obtained from two horses from Farm B [13]. The partial ORF5 nucleotide sequences were deposited into the International GenBank (LP01 DQ435439, LP02/R DQ435440, LP02/C DQ435441, LP02/P DQ435442, ROLP-ARG EU622862, RZ-LP-ARG EU622861 and KB-LP-ARG EU622860).

Dataset and Phylogenetic Analysis

The phylogenetic analysis was performed on the dataset built with 76 EAV sequences from different geographic regions (North America, Europe, Argentina, and South Africa). Eight of the sequences were reported in Argentina and the 68 additional sequences were obtained from the GenBank. Seven strains were isolated from seven semen samples collected from a donkey from South Africa (named as 'J2 to J7') (table 1). The region analyzed is located between nucleotides 11,296-11,813. The PRRSV-GXHP strain was used as outgroup (OG). Multiple sequence alignments were carried out using Clustal X version 1.92 software [20]. Sequence editing was performed with Bio-Edit version 7.05 [21]. Nucleotide diversity was estimated using MEGA 4 [22] and identity percent was calculated by SWAAP version 1.0.2 software [23]. The phylogenetic analysis was performed by neighbor joining (NJ), maximum parsimony (MP) and maximum likelihood (ML) algorithms. The NJ and ML trees were built by PAUP version $4.0 \mathrm{~b} 10$ [24]. To perform a phylogenetic analysis, the NJ and ML algo- 
rithms need the assumption of previous molecular evolution. The molecular evolution model was estimated using MrModeltest software, available at http://hiv-web.lanl.gov. The evolution model selected was Jukes-Cantor (JC). To perform a phylogenetic analysis using parsimony as optimality criterion, the dataset was analyzed using different strategies of weighting: equal weights, implied weights and differential TV/TS transformation cost. Two values of transversion/transition ratio (TV/TS) were explored. The different costs tested were: TV/TS: $2 / 1$. The internal gaps were treated as a 5th state, since they represent insertion-deletion events. The heuristic search procedure consisted of 1,000 replicates of random stepwise addition (RAS), and tree bisection and reconnection (TBR) branch swapping, using TNT software [25]. In order to assess the support of the identified groups, bootstrap [26] and jackknife tests [27] were performed. Both bootstrap and jackknife tests included 1,000 resampled matrices. For each resampled matrix, 1,000 RAS + TBR cycles were carried out. The phylogenetic analysis using the ML algorithm was performed using 100 RAS + TBR, the bootstrap test using 100 resample matrices, and each resampled matrix using 100 RAS + TBR cycles.

\section{Analysis of the Selective Pressures}

An analysis of the selective pressures was performed using the Tajima's test to establish evolutionary mechanisms that operate on the ORF5 of EAV strains, using DnaSP software [28] and Proseq version 3.0 software [29]. The additional analysis was performed using the non-synonymous $(\mathrm{dn})$ and synonymous $(\mathrm{ds})$ substitutions and their distances, based on the Nei-Gojobori method. The ratios of non-synonymous to synonymous substitutions (dn/ds) were calculated using SWAAP [23] and MEGA 4 [22].

\section{Results}

\section{Phylogenetic Analysis}

The identity percent between EAV sequences oscillated between 71.8 and $100 \%$. The nucleotide composition was: $\mathrm{T}(\mathrm{U})=34.3 \%, \mathrm{C}=23.3 \%, \mathrm{~A}=18.4 \%$ and $\mathrm{G}=24 \%$. Our dataset consisted of 511 sites, 246 of which were conserved sites and 234 parsimony sites. Parsimony analysis performed by heuristic searches using equal weight resulted in 67 1,399-step-long equally parsimonious trees. In the analysis under implied weights, 67 trees showing the same topologies were obtained. The strict consensus trees obtained for the most parsimonious trees made by equal and implied weights showed similar topologies. When transitions had a cost of 2/1 (TV/TS 2/1), 249 1,794 -step-long equally most parsimonious trees were obtained (fig. 1).

In this work, we built the bootstrap and jackknife trees using equal and implied weights and obtained similar results. Bootstrap (fig. 2) and jackknife trees showed similar topologies and presented minimal differences in support values. In these trees, two clades were identified; the
Table 1. Dataset of the 76 EAV sequences analyzed in this study, indicating name and place of origin

\begin{tabular}{|c|c|c|c|}
\hline Name & Country & Name & Country \\
\hline PRRSV-GXHP (OG) & China & KY77 & USA \\
\hline F5 & France & KY84 & USA \\
\hline F6 & France & KY93 & USA \\
\hline F7 & France & D84 & USA \\
\hline F8 & France & E85 & USA \\
\hline F9 & France & MT89 & USA \\
\hline F10 & France & VSB53 & USA \\
\hline F11 & France & $\mathrm{H} 20$ & Hungary \\
\hline F12 & France & $\mathrm{H} 21$ & Hungary \\
\hline F13 & France & $\mathrm{H} 22$ & Hungary \\
\hline F14 & France & $\mathrm{H} 23$ & Hungary \\
\hline F15 & France & $\mathrm{H} 24$ & Hungary \\
\hline F23 & France & $\mathrm{I} 13$ & Italy \\
\hline F24 & France & I14 & Italy \\
\hline F25 & France & I15 & Italy \\
\hline F26 & France & I16 & Italy \\
\hline J2-5941109-3 & South Africa & $\mathrm{I} 17$ & Italy \\
\hline J7-931125 & South Africa & $\mathrm{I} 18$ & Italy \\
\hline J6-940309 & South Africa & I19 & Italy \\
\hline J5-940309 & South Africa & $\mathrm{I} 20$ & Italy \\
\hline J4-931209 & South Africa & $\mathrm{R} 1$ & Europe (sd) \\
\hline J3-931209 & South Africa & $\mathrm{P} 1$ & Europe (sd) \\
\hline J2-931125 & South Africa & $\mathrm{A} 1$ & Europe (sd) \\
\hline RSA1 & South Africa & G1 & Europe (sd) \\
\hline RSA2 & South Africa & PLA00-1 & Poland \\
\hline RSA3 & South Africa & PLB01-1 & Poland \\
\hline RSA4 & South Africa & PLG02-1 & Poland \\
\hline RSA5 & South Africa & PLK02-8 & Poland \\
\hline RSA6 & South Africa & PLH05-1 & Poland \\
\hline RSA7 & South Africa & S544 & New Zealand \\
\hline RSA8 & South Africa & SWZ69 & Switzerland \\
\hline LP01 & Argentina & $\mathrm{S} 2$ & Sweden \\
\hline LP02/R & Argentina & $\mathrm{S} 3$ & Sweden \\
\hline LP02/C & Argentina & S4 & Sweden \\
\hline $\mathrm{LP} 02 / \mathrm{P}$ & Argentina & S5 & Sweden \\
\hline LT-LP-ARG & Argentina & S6 & Sweden \\
\hline RO-LP-ARG & Argentina & S7 & Sweden \\
\hline RZ-LP-ARG & Argentina & S-113 & Holland \\
\hline KB-LP-ARG & Argentina & & \\
\hline
\end{tabular}

PRRSV-GXHP was used as external group.

first clade, which showed high support values, was formed by eight strains, seven of which were isolated from seven semen samples collected from a donkey in South Africa - J2 to J7 [30, 31]. Our trees showed that the second clade presented four large groups, which had EAV sequences isolated from different geographic regions and isolated exclusively from Equus caballus. In this clade, we identi- 


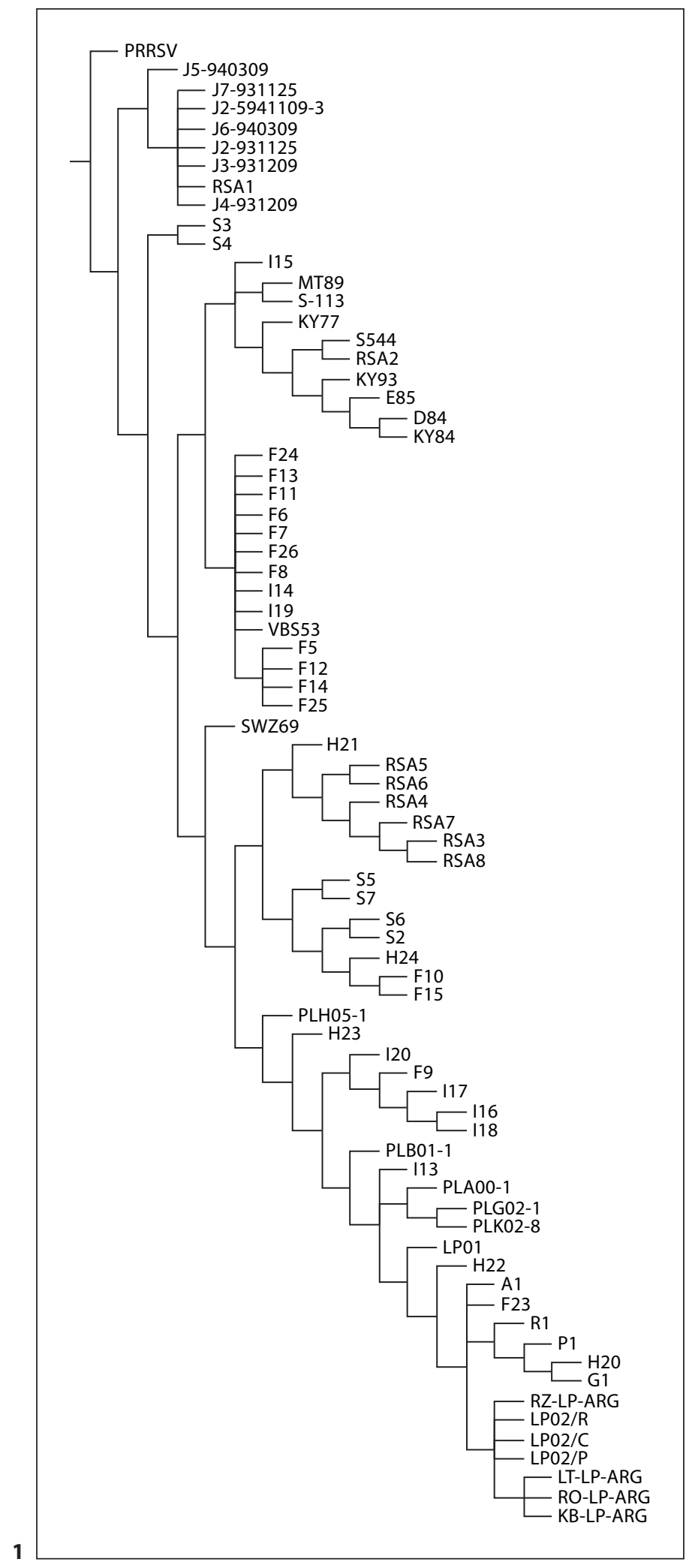

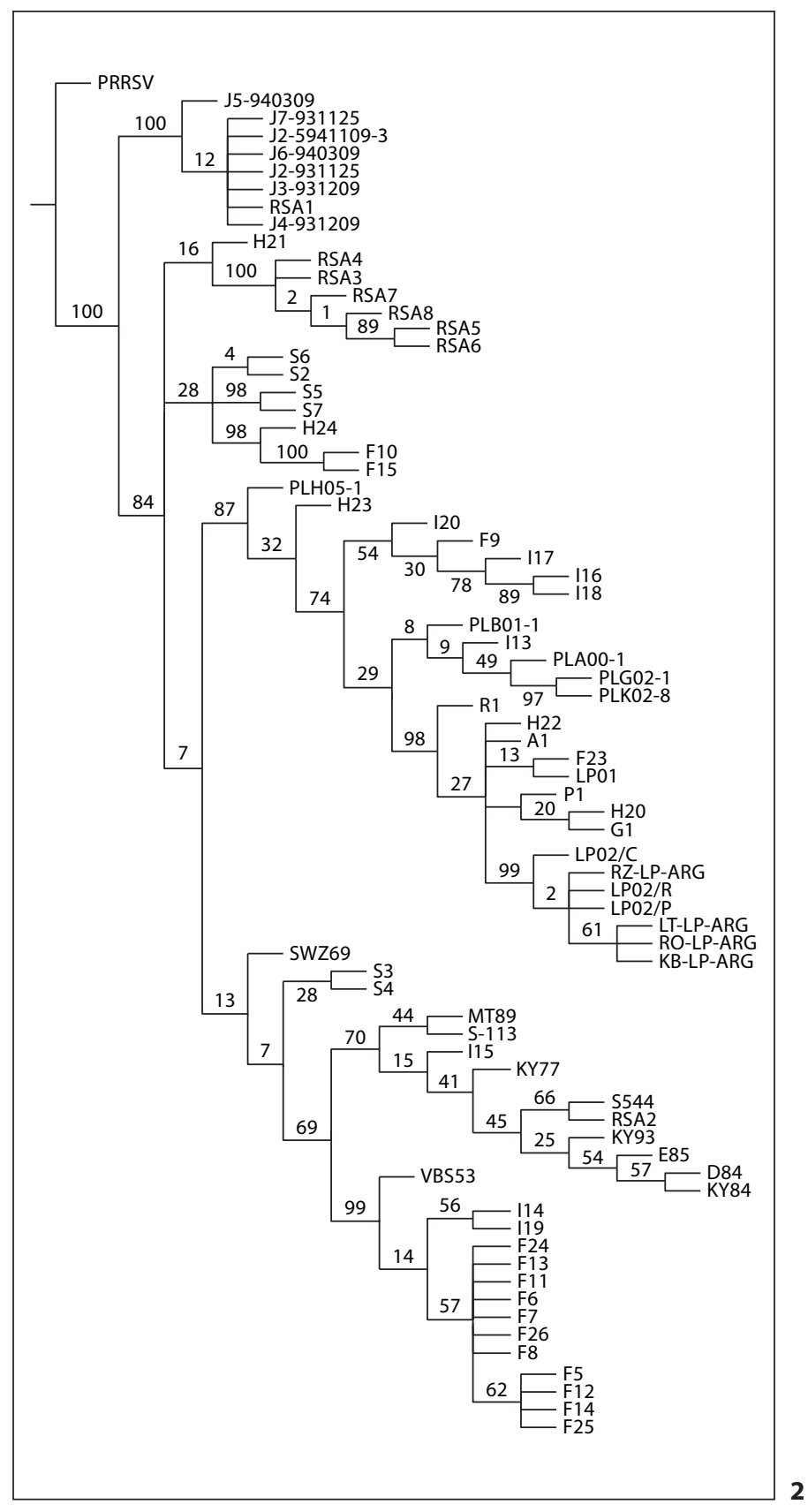

Fig. 1. Strict consensus tree of 76 sequences of EAV isolated from different geographical regions. This tree was obtained by parsimony as optimality criterion. Seven sequences of EAV were isolated from a donkey. PRRSV was used as external group.

Fig. 2. Phylogenetic tree obtained by the parsimony method from the analysis of the partial ORF5 of 76 sequences of EAV. Group support obtained with 1,000 bootstrapping replicates. Numbers above branches indicate support values. PRRSV was used as external group. 
Fig. 3. NJ tree obtained using JC molecular evolution model. A total of 76 sequences of EAV isolated from horses and a donkey were used in this study. PRRSV was used as external group.

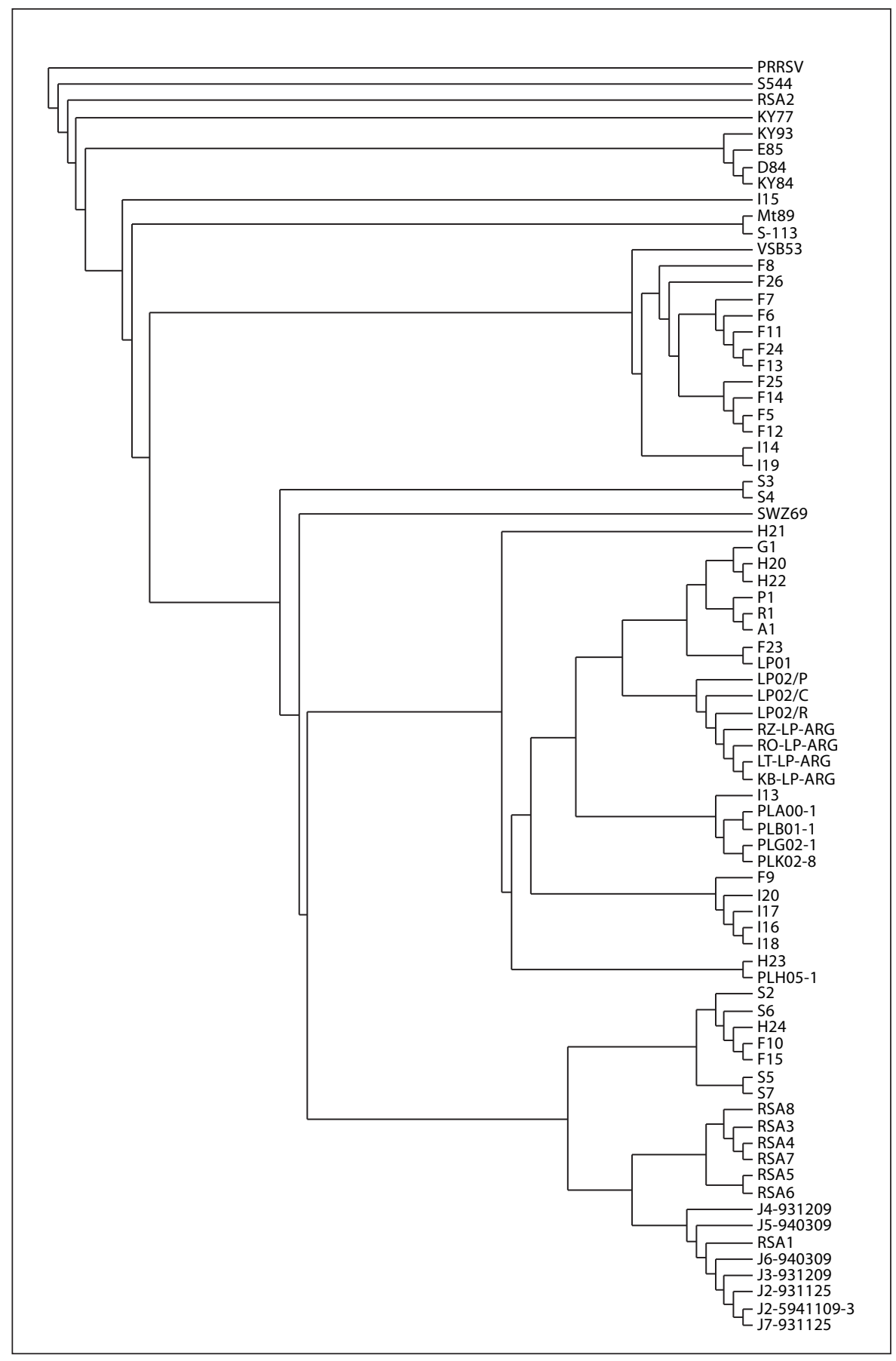

fied a group formed by the RSA3, RSA4, RSA5, RSA6, RSA7 and RSA8 sequences isolated from South Africa with a high bootstrap value $(=100)$, and another group formed by South American and European sequences, with a bootstrap value of 87 . In the latter group the monophyletic group was formed by the Argentinean sequenc- es LP02/C, LP02/R RZ-LP-ARG, LP02/P, LT-LP-ARG, RO-LP-ARG, and KB-LP-ARG, with a bootstrap value of 99. Another group, formed by the American (VSB53) and European sequences (I14, I19, F24, F13, F11, F6, F7, F26, F8, F5, F12, F14 and F25) with a high bootstrap value (=99), was identified in the second clade. 
The NJ tree (fig. 3) showed that we were able to identify the group formed by the Argentinean sequences LP02/C, LP02/R, RZ-LP-ARG, LT-LP-ARG, RO-LP-ARG and KB-LP-ARG. In addition, we identified that the South African strains RSA3, RSA4, RSA5, RSA6, RSA7 and RSA 8 were clustered together, whereas RSA 2 was the most basal strain with S544 strain, and that RSA1 was closely related to South African strains isolated from a donkey [31]. The American strains KY93, KY84, E85 and D84 were clustered together, whereas VSB53 was the most basal and remaining of the group conformed by F8, F26, F7, F6, F11, F24, F13, F25, F14, F5, F12, I14 and I19.

\section{Selective Pressures}

The Tajima's test applied on the partial ORF5 showed a $\mathrm{D}$ value of 1.73663 , with a confidence limit of $95 \%$ $(-1.737226$ to 1.86433$)$. The $\mathrm{D}$ value indicated that the EAV sequences analyzed follow a neutral evolution model. Calculation of the $\mathrm{dn} / \mathrm{ds}$ ratio was based on the number of synonymous and non-synonymous substitutions and its average was lower than $1(=0.38)$. Only purifying pressures - negative selection $(\mathrm{H} 0=\mathrm{DN}<\mathrm{DS})$ - were identified. The test applied in this work did not show other results to infer that would be positive pressures (where $\mathrm{H} 0=\mathrm{DN}>\mathrm{DS}$ ) in other regions of ORF5. From the hypothesis tested for evolution of the EAV partial ORF5, it was clear that the purifying selection played a role in the evolution of this ORF when comparing sequences taken from EAV sequences obtained from E. caballus and donkey.

\section{Discussion}

The aims of the present study were to increase our understanding about the genetic variability and the phylogenetic relationship between EAV worldwide sequences, mostly from Argentina, based on the analysis of the partial ORF5, to establish the origin of Argentinean sequences and to assess the evolutionary mechanism that operates on partial ORF5.

The sequence analysis of partial ORF5 of 76 EAV sequences isolated from different geographic regions revealed that the EAV sequences presented moderate variability between sequences. Other studies have shown that the French EAV isolates present high genetic diversity in this ORF [32].

In our study, we observed that the parsimony informative sites are coincident with the $\mathrm{N}$-glycosylation sites. Other authors have performed a phylogenetic analysis us-

Phylogeny of South American EAV Sequences ing different phylogenetic approaches. In 2007, Zhang et al. [32] performed a phylogenetic analysis using ORF5 and ORF3 by parsimony and NJ routines, and a bootstrap analysis was carried out on a 100-replicate dataset. This analysis showed two distinct groups: North American and European. The latter could be further divided in two subgroups: EU1 and EU2.

Although our analysis carried out by NJ and MP algorithms differed in some cases, all the approaches retrieved a similar grouping. The group formed by LT-LPARG, LP02 sequences, RO-LP-ARG, RZ-LP-ARG and KB-LP-ARG constitutes a monophyletic group, with a nearest common ancestor. Previous studies have shown that the Argentinean strains of the LP02 group form a monophyletic group, supported by a high bootstrap value [12].

Despite the results obtained in this study, we infer that the Argentinean sequences have a paraphyletic or polyphyletic origin. The paraphyletic origin of EAV was inferred by the scattered distribution of EAV sequences. According with the parsimony approach the paraphyletic group has a nearest common ancestor and some sequences of the ingroup (Argentinean EAV sequences). The analysis also showed that the LP01 sequence is separated from the remaining Argentinean sequences, suggesting that the strain has a different origin. However, the parsimony analysis identified two large clades: the first and the most basal of the tree, supported by a high bootstrap value, was formed by South African sequences isolated from a donkey and one sequence isolated from E. caballus, which allows inferring that interspecific transmission may have occurred; the second clade was formed by European, North American, South American and South African sequences isolated from E. caballus. The principal differences found among the results obtained by different phylogenetic approaches are based in the position on tree of groups retrieve under all the approaches.

In the NJ and ML trees, the group formed by RSA3, RSA4, RSA5, RSA6, RSA7 and RSA8 and that formed by J5-940309, J2-5941109-3, J6-940309, J2-931125, J3931209, RSA1, J4-931209 and J7-931125 appear at the terminal level of the trees, whereas in the parsimony tree, these groups appear at the basal level. According to our results, we may infer that the worldwide-circulating EAV isolated from E. caballus have a polyphyletic origin. The continuous transportation of equines between Europe and America may be the cause of the polyphyletic origin of the EAV strains, which confirms that the phylogenetic analysis allows establishing the origin of the EAV sequences. 
From the analysis of selective pressures of the ORF5 fragment obtained, we may infer that the fixation of point mutation might follow the neutral model evolution; however, we identified only purifying pressures that may be involved in the differentiation of EAV sequences. Purifying selection seems to be important as an evolutionary force driving the appearance of viruses.

\section{Acknowledgements}

The technical assistance of Ms. M.C. Mondragón and Mr. C. Leguizamón are highly acknowledged. This study was partially supported by PICT 2003 No. 1-13451 by the FONCyT.

\section{References}

1 Doll ER, Bryans JT, McCollum WH, et al: Isolation of a filterable agent causing arteritis of horses and abortion in mares. Its differentiation from the equine abortion (influenza) virus. Cornell Vet 1957;47:3-41

$\checkmark 2$ Cavanagh D: Nidovirales: a new order comprising Coronaviridae and Arteriviridae. Arch Virol 1997;142:629-633.

3 Snijder EJ: Arteriviruses; in Knipe DM, Howley PM (eds): Fields Virology. Philadelphia, Lippincott Williams \& Wilkins, 2001, pp 1205-1220.

4 Balasuriya UBR, Snijder EJ, Heidner HW, et al: Development and characterization of an infectious cDNA clone of the virulent Bucyrus strain of equine arteritis virus. J Gen Virol 2007;88:918-924.

$\checkmark 5$ Balasuriya UBR, Heidner WH, Hedges JF, et al: Expression of two major envelope proteins of equine arteritis virus as a heterodimer is necessary for induction of neutralizing antibodies in mice immunized with recombinant Venezuelan equine encephalitis virus replicon particles. J Virol 2000;74: 10623-10630

6 McCollum WH, Prickett ME, Bryans JT: Temporal distribution of equine arteritis virus in respiratory mucosa, tissues and body fluids of horses infected by inhalation. Res Vet Sci 1971;2:459-464.

7 Timoney P, McCollum W, Roberts A: Detection of the carrier state in stallions persistently infected with equine arteritis virus. Proc Am Assoc Equine Pract 1987;32:57-65.

$\checkmark 8$ Timoney PJ, McCollum WH: Equine viral arteritis. Vet Clin N Am Equine Pract 1993; 9:295-309.

9 González RJ, De la Sota M, Barrandeguy M, et al: Equine viral arteritis: serological survey in registered stallions in Argentina; in Proc. VIII Congress of the World Equine Veterinary Association, Buenos Aires, October, 2003, pp 194-195.

-10 Echeverría MG, Pecoraro MR, Galosi CM, et al: The first isolation of equine arteritis virus in Argentina. Rev Sci Tech 2003;22:10291033.
-11 Echeverría MG, Díaz S, Metz GE, et al: Genetic typing of equine arteritis virus isolates from Argentina. Virus Genes 2007;35:313320.

12 Metz GE, Serena MS, Martin Ocampos GP, et al: Equine arteritis virus: a new isolate from the presumable first carrier stallion in Argentina and its genetic relationships among the four reported unique Argentinean strains. Arch Virol 2008;153:2111-2115.

13 Metz GE, Serena MS, Díaz S, et al: Caracterización de secuencias del virus de la arteritis equina obtenidas directamente de muestras de semen de equinos seropositivos. Analecta Vet 2008;28:21-26.

14 Balasuriya UBR, Hedges JF, Smalley VL, et al: Genetic characterization of equine arteritis virus during persistent infection of stallions. J Gen Virol 2004;85:379-390.

15 Hornyak A, Bakonyi T, Tekes G, et al: A novel subgroup among genotypes of equine arteritis virus: genetic comparison of 40 strains. J Vet Med Sci B 2005;52:1-7.

16 Larsen LE, Storgaard T, Holm E: Phylogenetic characterization of the GL sequences of equine arteritis virus isolated from semen of asymptomatic stallions and fatal cases of equine viral arteritis in Denmark. Vet Microbiol 2001;80:339-346.

17 Stadejek T, Bjorklund H, Ros Bascunana C, et al: Genetic diversity of equine arteritis virus. J Gen Virol 1999;80:691-699.

18 Guthrie AL, Howell PG, Hedges JF, et al: Lateral transmission of equine arteritis virus among Lipizzaner stallions in South Africa. Equine Vet J 2003;35:596-600.

19 Timoney PJ: Equine viral arteritis; in Office International des Epizooties (ed): Manual of Standards for Diagnostic Tests and Vaccines, ed 4, 2000, pp 582-594.

20 Thomson JD, Gibson TJ, Pleuniak F, et al: Clustal X. Windows interface: a flexible strategy for multiple sequences alignment aided by quality analysis tools. Nuclei Acid Res 1997;24:4876-4882.
21 Hall TA: BioEdit: a user-friendly biological sequences alignment editor and analysis program for Window 95/98/NT. Nucl Acids Sym Ser 1999;41:95-98.

22 Tamura K, Dudley J, Nei M, et al: MEGA4: Molecular Evolutionary Genetics Analysis (MEGA) software version 4.0. Mol Biol Evol 2007;24:1596-1599.

23 Pride D: SWAAP: A Tool for Analyzing Substitution and Similarity in Multiple Alignments (http://www.bacteriamuseum.org/ SWAAP/SwaapPage.htm; distributed by the author), 2000.

24 Swofford DL: PAUP: Phylogenetic Analysis Using Parsimony (and Other Methods). Version 4. Sunderland, USA, Sinauer Assoc, 1998

25 Goloboff P, Farris S, Nixon K: 2008. TNT: a free program for phylogenetic analysis. Cladistics 2008;24:774-786.

26 Felsenstein J: Confidence limits on phylogenies: an approach using the bootstrap. Evolution 1985;39:783-791.

27 Farris JS Albert VA, Kallerersjo M, et al: Parsimony jackknifing outperforms neighborjoining. Cladistics 1996;12:99-124.

28 Librado P, Rozas J: DnaSP v5: a software for comprehensive analysis of DNA polymorphism data. Bioinformatics 2009;25:14511452.

29 Filatov DA: Proseq: A software for preparation and evolutionary analysis of DNA sequence data set. Mol Ecol Notes 2002;2:621624.

30 Paweska D, Volkmann DH, Barnard BJ, et al: Sexual and in-contact transmission of asinine strain of equine arteritis virus among donkeys. J Clin Microbiol 1995;33:32963299.

31 Stadejek T, Mittelholzer C, Oleksiewicz MB, et al: Highly diverse type of equine arteritis virus from the semen of a South African donkey. Acta Vet Hung 2006;54:263-270.

32 Zhang J, Miszczak F, Pronost S, et al: Genetic variation and phylogenetic analysis of 22 French isolates of equine arteritis virus. Arch Virol 2007;152:1977-1994. 